\title{
Comparing and contrasting development and reproductive strategies in the pupal hyperparasitoids Lysibia nana and Gelis agilis (Hymenoptera: Ichneumonidae)
}

\author{
Jeffrey A. Harvey
}

Received: 12 May 2006/ Accepted: 12 February 2007/Published online: 11 April 2007

(C) Springer Science+Business Media B.V. 2007

\begin{abstract}
In most animals, the optimal phenotype is determined by trade-offs in lifehistory traits. Here, I compare development and reproductive strategies in two species of solitary secondary hyperparasitoids, Lysibia nana and Gelis agilis, attacking pre-pupae of their primary parasitoid host, Cotesia glomerata. Parasitoid larvae of both species exploit a given amount of host resources with similar efficiency. However, adults exhibit quite different reproductive strategies. Both species are synovigenic, and female wasps emerge with no mature eggs. However, G. agilis must first host-feed to produce eggs, while L. nana does not host-feed but mobilizes internal resources carried over from larval feeding to initiate oogenesis. Further, G. agilis is wingless, produces large eggs, has a long life-span, and generates only small numbers of progeny per day, whereas these traits are reversed in L. nana. Given unlimited hosts, the fecundity curve in L. nana was "front-loaded," whereas in G. agilis it was depressed and extended over much of adult life. In L. nana (but not G. agilis), wasps provided with honey but no hosts lived significantly longer than wasps provided with both honey and hosts. Differences in the fecundity curves of the two hyperparasitoids are probably based on differing costs of reproduction between them, with the wingless G. agilis much more constrained in finding hosts than the winged L. nana. Importantly, L. nana is known to be a specialist hyperparasitoid of gregarious Cotesia species that pupate in exposed locations on the food plant, whereas Gelis sp. attack and develop in divergent hosts such as parasitoid cocoons, moth pupae and spider egg sacs. Consequently, there is a strong match between brood size in $C$. glomerata and egg production in $L$. nana, but a mismatch between these parameters in G. agilis.
\end{abstract}

Keywords Development - Cotesia glomerata - Hyperparasitoid - Lifetime reproductive success $\cdot$ Synovigenic $\cdot$ Trade-off

\section{J. A. Harvey $(\bowtie)$}

Netherlands Institute of Ecology (NIOO-KNAW), Centre for Terrestrial Ecology,

Boterhoeksestraat 48, 6666 ZG Heteren, The Netherlands

e-mail: j.harvey@nioo.knaw.nl 


\section{Introduction}

Understanding how limited metabolic resources are allocated for vital functions remains one of the foundations of both empirical and theoretical aspects of evolutionary ecology (Roff 1992; Stearns 1992). In most organisms, the optimal phenotype is determined by a trade-off in life-history traits, such as between early and late reproduction and between reproduction and survival (van Noordwijk and de Jong 1986; Lessells 1991; Rivero and West 2005). Consequently, an organism may invest more internal resources towards egg production early during adult life at the cost of a reduced life-span (Lessells 1991). A trade-off occurs when two traits are limited by the same resource, such as time or energy, because these can only be utilized once (Levins 1966; Lessells 1991). The relative importance of trade-offs in life-history traits critically depends on the quality of the habitat in which the organism lives, feeds and reproduces, in terms of resource availability (Pexton and Mayhew 2002; Holomuzki and Biggs 2006). Further, the risk of mortality experienced by the organism early during development may also influence metabolic investment towards maintenance or reproduction (Black and Dodson 1990; Riessen 1992; Vollrath and Parker 1992). Ultimately, life-history trade-offs may play an important role in shaping the structure of ecological communities (Bonsall et al. 2004).

Some of the best studies on life-history trade-offs and the importance of larval and adult feeding on reproduction have been conducted with parasitic wasps or "parasitoids" (Jervis and Kidd 1986; Chan and Godfray 1993; Collier 1995; Ellers et al. 1998; Rivero and West 2005). Parasitoids are insects that lay their eggs on or in another insect (the host), and the progeny develop by feeding on host tissues. Interactions between parasitoids and their hosts are usually highly intimate (Godfray 1994). For this reason, parasitoids exhibit developmental, physiological and morphological traits that are often highly specialized and correlated with ecophysiological characteristics of their hosts (Price 1972; Vinson and Iwantsch 1980; Jervis and Kidd 1986; Godfray 1994; Harvey and Strand 2002).

Parasitoids are classified according to the temporal production of eggs before and after adult emergence. Synovigenic parasitoids are species that emerge with only a fraction of their potential complement of mature eggs, whereas pro-ovigenic parasitoids emerge with a full egg complement and do not mature additional eggs during adult life (Jervis et al. 2001). The fitness of synovigenic parasitoids is largely dependent on the rate at which eggs can be matured after eclosion, the total number of eggs that can be produced (=potential fecundity), and the number of hosts that are successfully parasitized and that ultimately produced adult wasps (=realized fecundity). Host availability is in turn based on two interacting components, abundance and accessibility, and these parameters are strongly host-stage-dependent. For example, Price (1972) reported significant differences in life history and reproductive traits amongst guilds of parasitoids attacking different stages of the same host species, the sawfly Neodiprion swainei. Parasitoids attacking numerous, young larval hosts had high potential fecundities and short life-spans, whereas this situation was reversed for parasitoids attacking more scare pupal hosts.

Many parasitoids mobilize resources for egg production entirely from tissues carried over from larval feeding (Jervis and Kidd 1986; Ellers 1996; Heimpel and Collier 1996; Ellers et al. 1998; Harvey et al. 2001). Here, the adult parasitoid feeds on sources of carbohydrates, such as floral nectar, which contain sugars but are of low quality in terms of proteins (Boggs 1997). Carbohydrates in adult diet are used primarily for maintenance, and thus reduce the metabolic use of stored lipids for this purpose (Ellers 1996; Ellers et al. 1998, 2000; Ellers and Jervis 2003). Alternatively, in addition to nectar, the adults of many 
species of parasitoids also feed on high-quality resources, such as host body fluids and tissues, which are rich in proteins that are utilized directly for egg production (Jervis and Kidd 1986; Rivero and West 2005). Nutrients obtained from host-feeding thus enable the parasitoid to mobilize resources obtained from larval feeding primarily for maintenance purposes.

In parasitoids, as in other insects, the allocation of stored and incoming nutrients to reproduction influences the shape of a species' age-specific fecundity curve (Boggs 1997; Ellers and Jervis 2003). In holometabolous insects, with defined feeding and reproductive stages, differences in the shape of the curve may reflect concomitant differences in the importance of larval and adult feeding to reproduction (Ellers and Jervis 2003). When the quality of adult diet is low, resources obtained from larval feeding may be divided between reproduction and maintenance, and as a consequence the fecundity curve is skewed towards early reproduction (more pro-ovigenic) and reduced longevity (Price 1972; Boggs 1997). In contrast, when the quality of adult diet increases, this may free resources obtained from larva feeding to be used more towards maintenance and resources obtained from adult feeding to be invested more into egg production (Boggs 1997). In this situation, the age-specific fecundity curve is dampened but may be extended over a significant portion of adult life (more synovigenic), and longevity is greatly extended.

This study compares and contrasts host utilization, development, and reproductive strategies in two species of solitary, secondary idiobiont hyperparasitoids that attack fully cocooned (pre)pupae of the same primary parasitoid host. Cotesia glomerata L. (Hymenoptera: Braconidae) is a gregarious primary endoparasitoid that attacks young larvae of cabbage white butterflies, including Pieris brassicae L. (Lepidoptera: Pieridae). Lysibia nana Gravenhorst (Hymenoptera: Ichneumonidae) is a fully winged, sexually reproducing obligate hyperparasitoid that only attacks closely related primary hosts in the braconid subfamily Microgastrinae that pupate in exposed locations, including $C$. glomerata. Adult L. nana females do not host-feed, and thus rely on sources of nectar in their habitat. Gelis agilis Fabricius (Hymenoptera: Ichneumonidae) is a wingless, asexually reproducing facultative hyperparasitoid of several Cotesia species that is also potentially a primary parasitoid of many other hosts in nature. For example, in addition to parasitoid cocoons, G. agilis and other Gelis species are known to attack such evolutionarily divergent hosts such as moth pupae and even spider egg sacs (Bezant 1956; Schwarz and Boriani 1994; van Baarlen et al. 1996; Cobb and Cobb 2004; Toth and Lucas 2005). In addition to nectar sources, adult G. agilis females obtain extra resources by feeding on host fluids.

The main aims of the study are (a) to determine if the larvae of both hyperparasitoids exploit host resources with similar efficiency, (b) to compare daily fecundity schedules (fecundity curves) and lifetime reproductive success of the two species provided with excess numbers of hosts in laboratory experiments, and (c) to compare other reproductive traits, including egg production and size in L. nana and G. agilis. Based on the results, I argue that option sets for resource exploitation by parasitoid larvae exploiting a given host stage are likely to be limited, resulting in similar patterns for size and development time in hosts of a given mass at parasitism. However, I also suggest that the presence or absence of wings in both hyperparasitoid species may affect the spatial dynamics of their searching patterns. This may account for trade-offs in the allocation of resources to reproduction and longevity in the two species, and may also explain differences in their host ranges. 


\section{Methods and materials}

Insects

Hosts and parasitoids were maintained at $25 \pm 2^{\circ} \mathrm{C}$ under a 16:8 h L:D regime. Cultures of C. glomerata and P. brassicae were obtained from insects reared at Wageningen University (WUR), the Netherlands, and which were originally collected from agricultural fields in the vicinity of the University. All P. brassicae larvae used in these experiments had been maintained on Brassica oleracea var. Cyrus (Brussels sprouts) at WUR. In these experiments, $P$. brassicae larvae were maintained on $B$. oleracea plants that originated from seeds from plants growing along a road near the Institute of Ecology at Driel, the Netherlands.

C. glomerata were reared according to the protocol described in Harvey (2000). Adult female wasps oviposit 10-40 eggs into first (L1) to third (L3) instars of P. brassicae. During their development parasitoid, larvae feed primarily on host hemolymph and fat body and emerge from the host caterpillar late during its final instar. After emergence, wasp larvae immediately spin cocoons on the host plant adjacent to the host, which perishes within a few days.

L. nana was originally obtained from cocoons of $C$. glomerata recovered from leaves of B. napus growing in a garden plot adjacent to the Institute of Ecology, Heteren, the Netherlands. It is known to attack several closely related gregarious endoparasitoid species in the higher Microgastrinae that pupate in exposed locations on the primary host foodplant, including $C$. glomerata. G. agilis was also originally obtained from cocoons of $C$. glomerata placed into the field adjacent to the Institute. Little is known about its biology and ecology (but see van Nouhuys and Hanski 2000; Harvey and Witjes 2005).

Like many ectoparasitic idiobionts, adult females of both hyperparasitoids perforate the host cocoon with their ovipositor and inject permanently paralyzing venom into the prepupa or pupa. Following envenomation, the wasps lay a single egg on the moribund host. After the parasitoid egg hatches, the larva perforates the host cuticle with its mandibles and imbibes hemolymph, but as it grows it begins attacking other tissues indiscriminately and eventually consumes the entire host, pupating within the cocoon of $C$. glomerata. In culture, L. nana and G. agilis were maintained exclusively on 1-2-day-old pupae of $C$. glomerata. After emergence, hyperparasitoids were kept in large (20 cm diameter) Petri dishes at $10^{\circ} \mathrm{C}$. Complete rearing methods of both species are described in Harvey and Witjes (2005).

\section{Experimental protocol}

\section{Relationship between host mass and adult hyperparasitoid mass}

Larvae of $P$. brassicae were parasitized in the first instar (L1) by females of $C$. glomerata by presenting individual larvae to parasitoids at the end of a brush in plastic vials. Parasitism was ensured by allowing wasps to sting hosts for at least $10 \mathrm{~s}$, which enables a full brood to be laid (Harvey 2000). Parasitized caterpillars were immediately placed in large rearing cages $(1 \mathrm{~m} \times 60 \mathrm{~cm} \times 60 \mathrm{~cm})$ containing four $B$. napus plants. These were refreshed once every few days as required.

Between 12 and 24 h prior to parasitoid emergence, larvae of $P$. brassicae spin a silken mat on the surface of a leaf or inside the rearing cage. These larvae were collected and 
placed individually into Petri dishes $(10 \mathrm{~cm}$ diameter). Upon larval parasitoid emergence from the host (prior to pupation), separate broods of $C$. glomerata cocoons were collected and separated carefully using a pair of forceps and a cecum. Cocoons of C. glomerata within broods were then numbered and weighed individually on a Mettler-Toledo MT5 Electrobalance (accuracy $\pm 1 \mu \mathrm{g}$ ). Two rows of five cocoons that had been pre-weighed were laid down in a Petri dish and attached to the bottom of the dish with a small drop of honey. A L. nana female was introduced into the Petri dish and allowed to forage for $24 \mathrm{~h}$. It was then removed and returned to the main culture. The cocoons were also removed from the dishes and placed in labeled 2-1 plastic vials until adult emergence of the hyperparasitoids. Newly emerged wasps were anesthetized using $\mathrm{CO}_{2}$ and weighed on the Mettler microbalance. Adult wasps were sexed by visually checking for the presence or absence of an ovipositor on the wasps. The same experiment was repeated with G. agilis, except that in this case females were offered single rows of five cocoons, and all emerging adults were females. For $L$. nana, $n=141$ wasps ( 105 males, 36 females) from 15 broods of C. glomerata; for G. agilis $n=14$ wasps from 10 cocoon broods.

\section{Egg production, longevity, and lifetime reproductive success in L. nana and G. agilis}

Newly emerged wasps of both species were weighed on the microbalance, placed individually in Petri dishes (12 cm diameter) and provided ad libitum with honey. A small ball of cotton soaked in water was also added to ensure that the honey remained partly in solution (and accessible to the wasps, which cannot imbibe dry honey with their mouthparts). To determine egg loads, separate cohorts of wasps were maintained as described above. Beginning on the day of eclosion, 10 wasps (of L. nana) and 5 (of G. agilis) were dissected in a drop of water on a glass slide using two pairs of forceps and a cecum, and the number of mature (=ovulated, fully chorionated) eggs in their ovaries counted. For L. nana, this process was repeated daily over the first 5 days and then on days 10 and 20 posteclosion. In G. agilis, the same experiment was repeated (as with L. nana) except that there were two treatments: in the first, wasps were deprived of hosts, their ovaries removed, and eggs (if any) counted on days $0,2,4,6,10,15$, and 20 days post-eclosion; in the second, wasps were provided with 10 host cocoons for the first $24 \mathrm{~h}$ only post-eclosion and were then dissected from day 2 onwards and the number of eggs counted (as in the control).

Hyperparasitoids were weighed at eclosion and thereafter maintained in Petri dishes (as described above) with honey and water. Beginning 3 days after eclosion, cocoons of $C$. glomerata that were $<48$-h old (containing young pupae) were presented to individual hyperparasitoids of both species. It was important to evaluate how many cocoons of $C$. glomerata would need to be provided to both hyperparasitoids in order to ensure that cocoon number was not a limiting factor in daily oviposition and reproductive success. This was estimated on the basis of ovary dissections and the results of a previous study (Harvey and Witjes 2005). Moreover, irrespective of host density, pilot experiments revealed adults of $G$. agilis will not parasitize more than three to four cocoons per day. Typically, the wasp kills one or two hosts daily for host-feeding purposes, parasitizes one to four other hosts and ignores other cocoons within a brood of $C$. glomerata. In contrast, L. nana does not host-feed and begins to mature eggs on the second to third day after eclosion and, when hosts are unavailable, produces about 10-15 eggs daily for a few days until the ovaries reach their storage capacity (Harvey and Witjes 2005). Based on these criteria, for the lifetime reproductive success experiments $L$. nana ( $n=10$ wasps) was provided continually with 20 cocoons/day until wasp death whereas G. agilis $(n=10$ 
wasps) was provided with 10 host cocoons/day (until 50 days) and five cocoons/day thereafter until wasp death. At the end of each 24-h period, cocoons were removed and placed in plastic vials with information on the date, wasp species and number written onto adhesive labels on the vials. Data on the fate of all of the cocoons were determined (producing an adult hyperparasitoid, parasitoid, or precocious death). Longevity of L. nana and G. agilis was also measured by recording the number of days between adult eclosion and death with and without host access. In order to determine if host-feeding also influences longevity in G. agilis, separate cohorts of wasps ( $n=10$ for each treatment) were reared from eclosion on the following diets: honey and water, hosts and water, water only, hosts only, and host fluids (hosts were removed from cocoons and mashed using a small spatula, and this was diluted in a small droplet of water and presented to wasps).

\section{Statistical analyses}

The relationship between host cocoon mass in $C$. glomerata and adult body mass in $L$. nana and G. agilis was compared through regression analyses. Hyperparasitoid longevity was analyzed using two-way ANOVA, with species and host access (with or without hosts) as factors. For G. agilis, longevity was compared by means of one-way ANOVA. Multiple comparisons were made by means of Tukey's LSD tests. Mean total fecundity in L. nana and G. agilis was compared using a Student's $t$ test. The relationship between adult body mass and reproductive success was compared through regression analyses.

\section{Results}

Adult body size in $L$. nana males $\left(F_{1,13}=73.65, P<0.001\right)$ and females $\left(F_{1,9}=304.76\right.$, $P<0.001)$, as well as in $G$. agilis females $\left(F_{1,11}=45.39, P<0.001\right)$, varied significantly with host size. Host cocoons that were heavier at parasitism produced larger adult hyperparasitoids than smaller cocoons (Fig. 1). The larvae of both species appeared to exploit a given amount of host mass with quite similar efficiency (Fig. 1).

Fully ovulated eggs of the two hyperparasitoids are shown in Fig. 2. Eggs of L. nana are considerably smaller than eggs of G. agilis, which produces eggs approaching $1 \mathrm{~mm}$ in length. Accordingly, maximum egg loads in the two species differed significantly over the course of the first 20 days of adult life. Both species are completely synovigenic, and emerge with no fully matured eggs (Fig. 2b). However, in the absence of hosts, L. nana begins maturing eggs soon after eclosion and attains a maximum complement of eggs in the ovaries by about the 5th day post-eclosion (Fig. 2b). In contrast, G. agilis produces no eggs unless female wasps are supplied with hosts. Wasps that were supplied with hosts over the first $24 \mathrm{~h}$ of adult life produced between one and three mature eggs and this total remained fairly constant over the first 20 days (Fig. 2b).

Hyperparasitoid longevity varied significantly between $L$. nana and $G$. agilis $\left(F_{1,48}=62.16, P<0.001\right)$ and with access to hosts or under host-deprivation $\left(F_{1,48}=7.05\right.$, $P=0.011)$ but the interaction between these parameters was not significant $\left(F_{1,48}=0.03\right.$, $P=0.86$ ). Female hyperparasitoids that were provided with hosts had on average significantly shorter life-spans than wasps deprived of hosts, and G. agilis lived on average up to 3 weeks longer than L. nana (Fig. 3a). In G. agilis, there was also a significant difference in hyperparasitoid longevity between different food-host access treatments $\left(F_{4,52}=80.60\right.$, $P<0.001$ ). Wasps provided with honey and water (with or without hosts) lived consid- 


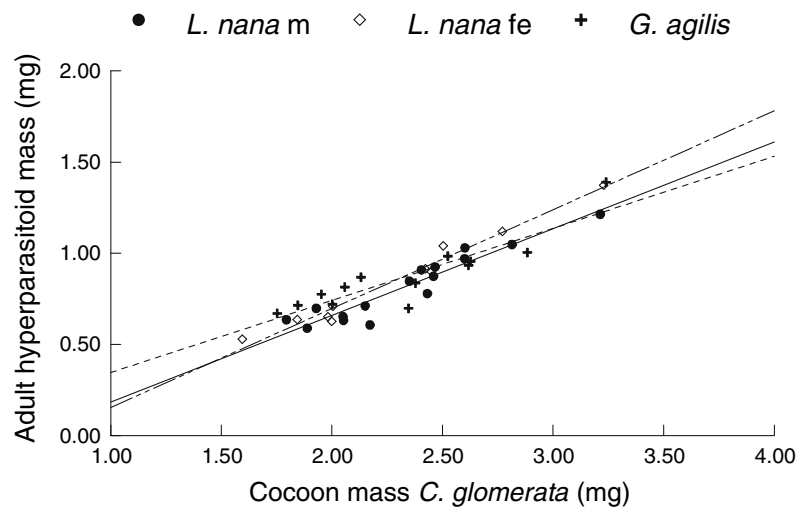

Fig. 1 Relationship between cocoon mass (in $\mathrm{mg}$ ) of $C$. glomerata and emerging adult male and female mass in L. nana and female mass in G. agilis. L. nana male wasps (closed circles, solid line): $y=0.49 x-$ $0.33, R^{2}=0.85 ;$ L. nana female wasps (open diamonds, short- and long-dashed lines): $y=0.54 x-0.39$, $R^{2}=0.97 ; G$. agilis female wasps (crosses, dashed line): $y=0.39 x-0.05, R^{2}=081$. Sample sizes are: $L$. nana males, mean mass from 15 cocoon broods (total of 105 wasps); L. nana females, mean mass from 10 cocoon broods (total of 41 wasps); G. agilis females, mean mass from 10 cocoon broods (total of 14 wasps)

Fig. 2 (a) Mature, ovulated egg of G. agilis (top) and two mature, ovulated eggs of L. nana (below). Scale $=1 \mathrm{~mm}$. (b) Mature egg loads of adult female L. nana (closed diamonds) and G. agilis (open circles) at various times over 20-days post-eclosion. Female G. agilis that were deprived of hosts produced no eggs (not shown). This species must host-feed to produce eggs, therefore five cocoons were provided to wasps over the first $24 \mathrm{~h}$ of adult life (black bar). Line bars represent standard error of the mean. Sample size: 10 wasps/day for L. nana, and five wasps/day for G. agilis

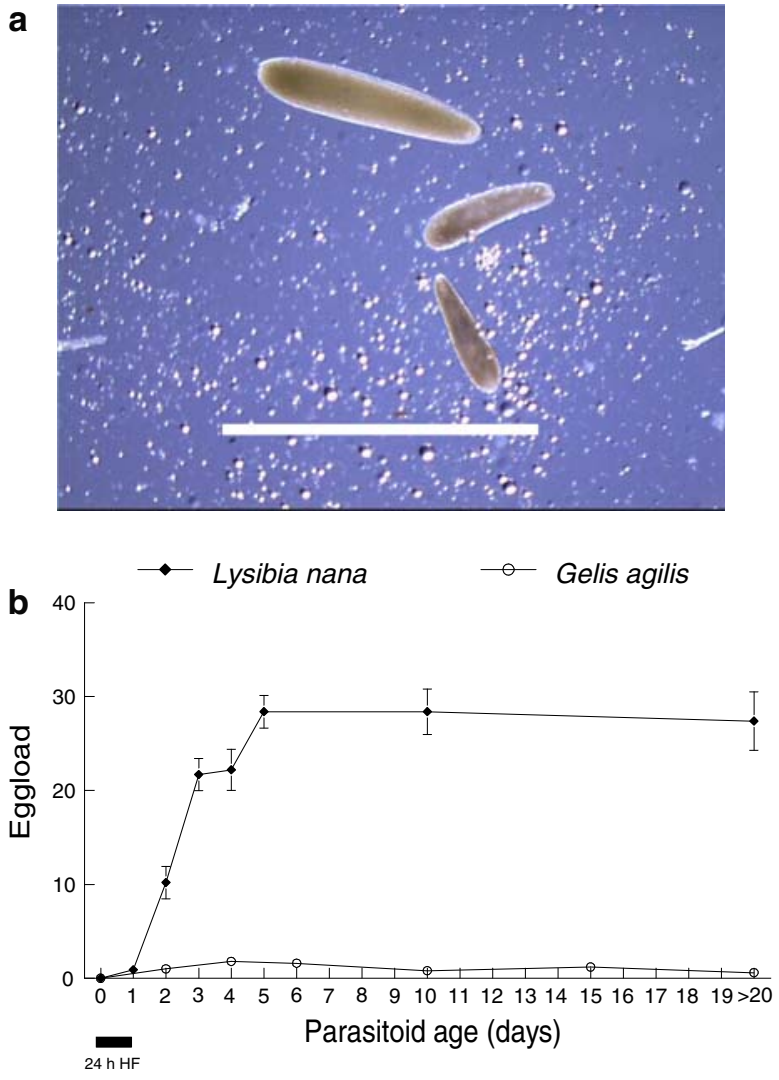


Fig. 3 (a) Mean adult longevity (in days) of female G. agilis and $L$. nana when provided with hosts throughout the course of adult life (filled bars) and when deprived of hosts (hatched bars). Bars with the different letters are significantly different $(P<0.05$, Dunnett's HSD test). Sample sizes (number of wasps): $G$. agilis without hosts $=15$, with hosts $=10 ;$ L. nana without hosts $=19$, with hosts $=10$. $(\mathbf{b})$ Mean adult longevity (in days) of different cohorts of G. agilis under the following food/host access treatments: constant honey and water (black bar), constant honey, water, and hosts (open bar), constant water only (vertical hatched bar), host fluids only (grey bar), and hosts only (angled hatched bar) (partial data repeated from a). Sample sizes: honey, water $=15$, all other treatments $=10$. Line bars: standard error of the mean
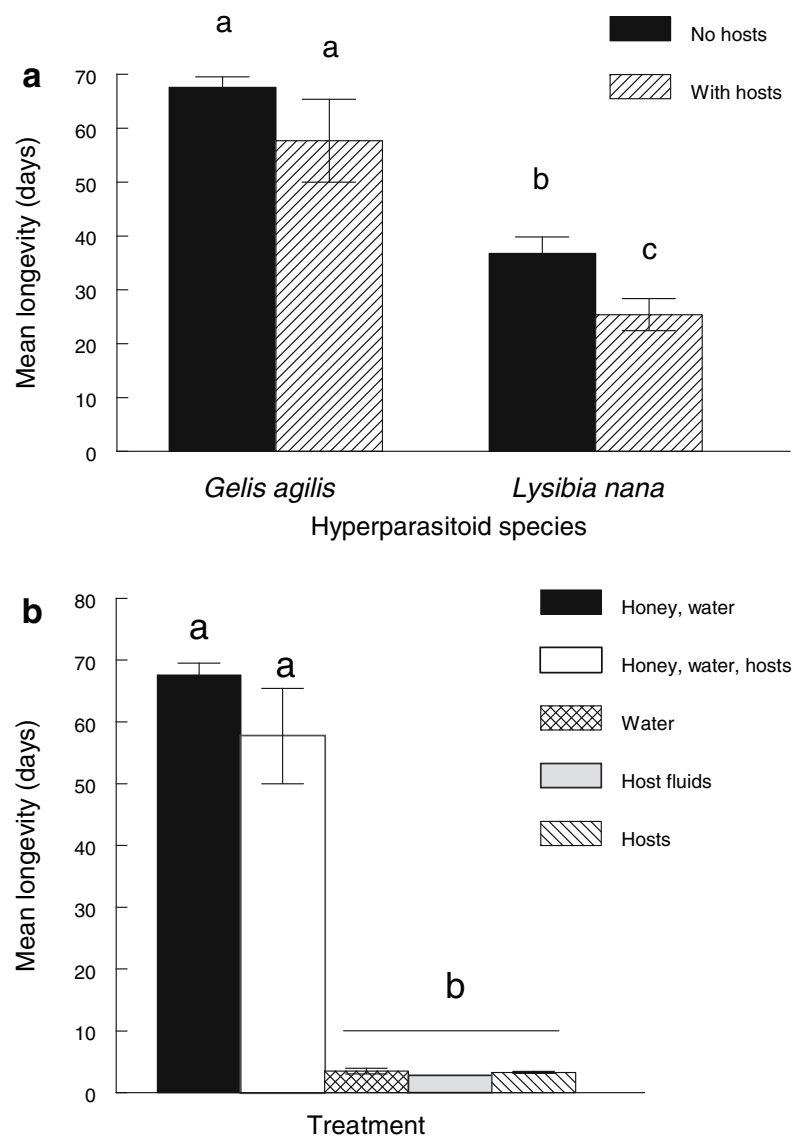

erably longer than wasps provided with only water, hosts, or host fluids in the absence of honey (Fig. 3b). In the case of G. agilis, two wasps provided with hosts and honey lived almost 100 days.

Adult body size of L. nana and G. agilis wasps used in fecundity experiments was not significantly different $\left(t_{18}=1.31, P=0.21\right)$. In fact, G. agilis wasps (mean $=1.301 \mathrm{mg}$ ) were marginally larger than $L$. nana wasps (mean $=1.092 \mathrm{mg}$ ). Lifetime reproductive success was, however, significantly higher in L. nana than in G. agilis $\left(t_{18}=6.94\right.$, $P<0.001)$. There was actually little overlap in the minimum number of offspring produced by the least fecund $L$. nana female (70) and the maximum number of offspring produced by the most fecund $G$. agilis female (72). On average, L. nana females produced more than twice as many progeny as G. agilis females (Fig. 4a). However, the age-specific fecundity curves of the two species also differed markedly. Given unlimited host access, progeny production in L. nana was heavily skewed over the first several days, and peaked as early as the second day, declining steadily thereafter. Many wasps experienced fairly long periods of post-reproductive survival, in some instances exceeding the period of oviposition (Fig. 4b). In contrast, progeny production in G. agilis gradually increased over the first 4-5 days of host access and then remained fairly constant over the following 2 weeks. Only after this time did daily fecundity begin to decline, although a few wasps were still producing offspring more than 10 weeks after emergence (Fig. 4b). 
Fig. 4 (a) Mean lifetime reproductive success of $L$. nana and $G$. agilis provided with constant honey, water, and excess hosts. Sample size $=10$ wasps for each species. (b) Daily patterns of progeny production for $L$. nana and $G$. agilis provided with constant honey, water, and excess hosts. Line bars: standard error of the mean. Sample size as in $\mathbf{a}$
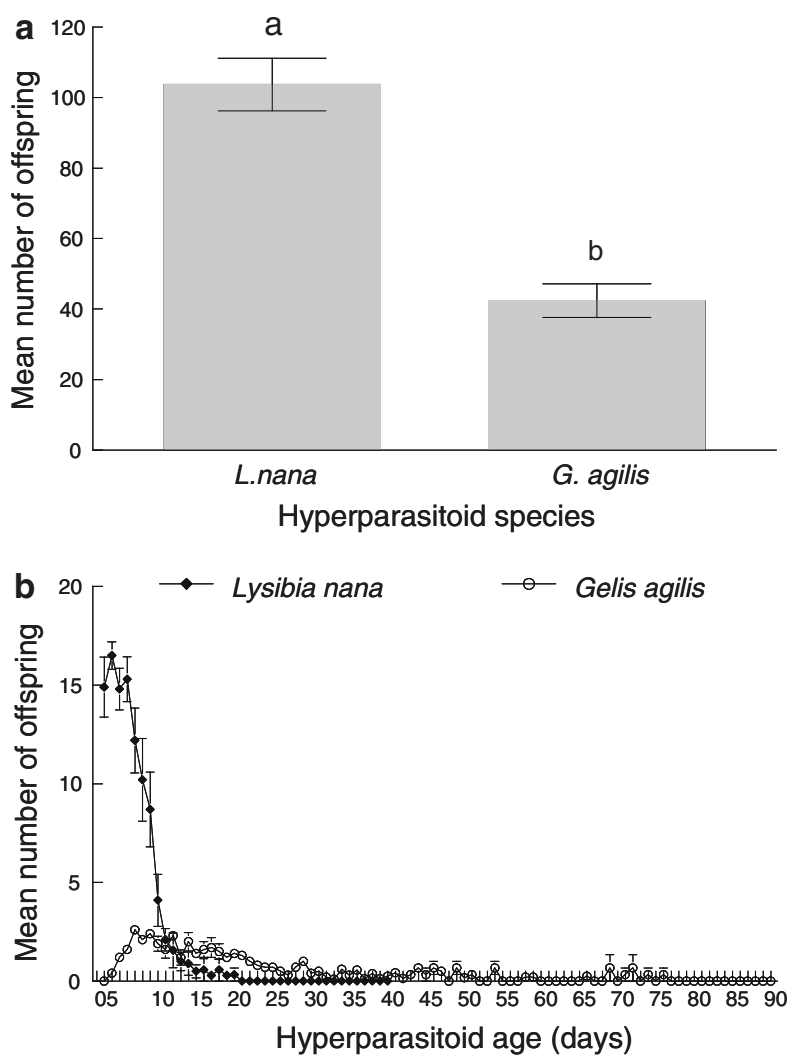

The relationship between adult body size and total progeny production was significant in L. nana $\left(F_{1,8}=5.72, P<0.05\right)$ but not in $G$. agilis $\left(F_{1,8}=1.26, P>0.05\right)$. Larger females of $L$. nana were capable of producing more than 130 offspring, whereas small females generally produced less than 100 offspring (Fig. 5a). However, the relationship between body size and progeny production in G. agilis was significant over the first 20 days of adult life $\left(F_{1,8}=8.73, P=0.01\right.$; Fig. $\left.5 b\right)$. This is the period in which $G$. agilis produces most progeny. Longevity in both hyperparasitoids was not influenced by body size with and without hosts: $L$. nana control $\left(F_{1,17}=0.73, P>0.05\right)$; $L$. nana with hosts $\left(F_{1,8}=0.02\right.$, $P>0.05) ; G$. agilis control $\left(F_{1,3}=1.48, P>0.05\right) ; G$. agilis with hosts $\left(F_{1,8}=2.68\right.$, $P>0.05)$.

\section{Discussion}

In G. agilis and L. nana, significant convergence was found in some biological traits, whereas in other respects these parasitoids were very different. For a given host (cocoon) mass at parasitism, adults of L. nana and G. agilis were of approximately the same size. The size of adult hyperparasitoids was correlated with the mass of the host cocoon, although the relationship was somewhat stronger in L. nana. Both species exhibited remarkable efficiency in converting host tissues into hyperparasitoid tissues. A previous 
Fig. 5 (a) Relationship between adult hyperparasitoid size and lifetime reproductive success in L. nana (closed triangles) and $G$. agilis (open circles) provided with constant honey, water, and excess hosts. Regression equation: L. nana, $y=0.01 x+0.20, R^{2}=0.42 ; G$. agilis, $y=0.01 x+0.89$, $R^{2}=0.14$. Regression line provided for L. nana data only because this was significant $(P<0.05$; see “Results"). (b) Relationship between adult body size and lifetime reproductive success in G. agilis provided with constant honey, water, and excess hosts over the first 20 days of adult life. Regression equation: $y=14.31 x+11.89, R^{2}=0.52$. Sample size as in Fig. 4
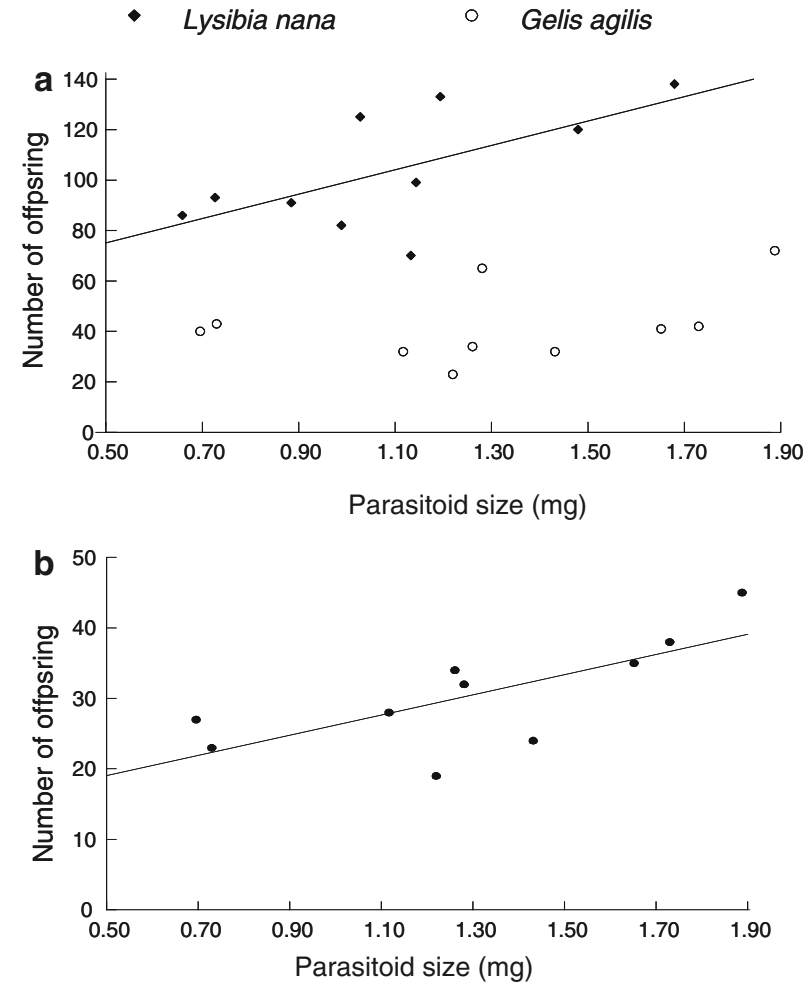

study with $L$. nana reported that hyperparasitoid adults emerging from host cocoons of a given mass at parasitism were up to $98 \%$ as large as $C$. glomerata adults that had been allowed to develop in cocoons of a comparable size (Harvey et al. 2006). Consequently, hyperparasitoids are likely to be amongst the most efficient organisms in the animal kingdom at utilizing a given amount of food resources (Harvey et al. 2006).

In contrast with development, highly significant interspecific differences were observed in daily patterns of progeny production, lifetime reproductive success, and other biological and reproductive traits in the two hyperparasitoids. When provided constantly with a source of adult nutrition (honey solution), G. agilis females lived almost twice as long as $L$. nana females. G. agilis is wingless and exhibits ant-like behavior while foraging on the ground, whereas L. nana is fully winged and is an efficient flier. Price (1972) suggested that the loss of wings in parasitoid taxa such as Gelis spp. is an efficiency measure, allowing the parasitoids to utilize the thoracic cavity to store fat body (used for maintenance) that in winged species is taken up primarily by wing musculature.

Unlike L. nana, which does not host-feed and mobilizes metabolic proteins for egg maturation obtained during larval development, G. agilis can only initiate oogenesis when proteins are obtained exogenously through destructive host-feeding behavior (Jervis and Kidd 1986). In addition to proteins in host fluids that are mobilized for the production of germinal tissues (eggs), host hemolymph is also known to contain sugars, such as trehalose and sucrose, that slow the metabolic use of fat body that is mobilized for somatic tissues (maintenance) (Jervis and Kidd 1986; Ellers et al. 1998; Ueno 1999; Giron et al. 2002). However, longevity in G. agilis was only extended when honey solution was available, 
revealing that host fluids play little or no role in metabolic maintenance of this species. Studies with other host-feeding parasitoid species have reported that longevity is extended when female wasps are allowed to feed on both host fluids and honey or sugar solution (Heimpel et al. 1997; Lauziere et al. 2000), whereas in other species there is no apparent benefit of host-feeding on longevity (Giron et al. 2004; Rivero and West 2005). Sources of carbohydrates in the local environment, such as floral nectar, are thus likely to be an important factor in sustaining populations of many host- and non-host-feeding parasitoids (Wäckers 2001).

In L. nana (but not G. agilis), parasitoid longevity was lower in cohorts of wasps provided with hosts and honey than in wasps that were deprived of hosts. This finding supports the hypothesis of trade-offs between life-history characters, and suggests that there were constraints on the efficiency with which metabolic resources could be acquired and allocated to vital physiological functions in L. nana (Reznick 1985). The differing effects of host access on longevity in the two species may be based on the differences in strategies they employ to obtain resources for maintenance and reproduction. This suggests that internal storage sites in L. nana are rapidly depleted when resources are allocated to both metabolic activity and reproduction. Alternatively, in G. agilis host-feeding enables resources obtained from larval feeding to be used primarily for maintenance and resources obtained from host-feeding to be used for reproduction. Some studies with parasitoids have found evidence of a trade-off between reproduction and survival (Hohmann et al. 1989; Orr and Boethel 1990), whereas others have not (Bai and Smith 1993; Harvey et al. 2001). Thus, the costs of physical activity and reproduction in parasitoids are likely to be association-specific.

Maximum daily progeny production and total reproductive success was much higher in L. nana than in G. agilis, but temporal patterns of progeny production also differed significantly between the two species. Both species are completely synovigenic, but L. nana begins rapidly maturing eggs soon after eclosion. Age-specific fecundity in L. nana exhibited a short peak early in life and then declined sharply, whereas the fecundity curve of G. agilis was greatly dampened, and offspring were produced over a much longer period of adult life. Irrespective of host (cocoon) availability, G. agilis usually only produced two to four progeny per day, which was also reflected in ovary dissections of wasps that were initially allowed to host-feed but subsequently deprived of hosts. On the other hand, over the first few days $L$. nana was able to parasitize more than 15 cocoons per day. In nature, $C$. glomerata produces single broods that typically vary in number between 10 and 40 wasps per brood (Harvey 2000). Therefore, an individual female of $L$. nana is probably able to parasitize a much larger proportion of a single C. glomerata brood than is G. agilis (Schwarz and Shaw 2000).

These results have demonstrated that, even amongst closely related species attacking a common host type, there may be strong convergence in some evolved characters (e.g., development) and a strong divergence in others (e.g., reproductive biology). In parasitoid larvae, option sets for exploiting host resources are limited by the simple fact that even a slight divergence from a given strategy can result in a significant reduction in fitness or even precocious death (Mackauer and Sequiera 1993). Idiobiont parasitoids such as $L$. nana and G. agilis attack resources that are fixed at parasitism and that gradually decline in quality as the host ages. Under these circumstances, the optimal strategy is for the parasitoid larvae to develop at their maximal rate. In contrast, koinobiont parasitoids develop in hosts that continue feeding and growing during much of the interaction. Because host resources are dynamic, the optimal development strategy of the parasitoid depends critically on the host's initial size and on its growth rate, as well as on its susceptibility to other 
natural enemies such as insect predators (Harvey and Strand 2002). Consequently, development strategies amongst koinobionts appear to be much more flexible than is the case with idiobionts (Mackauer and Sequeira 1993).

Reproductive strategies in parasitoids represent an adaptive response to a range of selection pressures, in particular host abundance, and accessibility (Price 1972; Pexton and Mayhew 2002). Because it is wingless, G. agilis can only exploit a relatively small area during its lifetime. Under these conditions, selection should favor an expansion in the parasitoid's host range, because suitable hosts will be difficult to find. Different species of Gelis are known to parasitize a wide variety of arthropod hosts, including moth pupae and spider egg sacs in addition to parasitoid cocoons, supporting this hypothesis (Bezant 1956; van Baarlen et al. 1996). It is therefore quite likely that $G$. agilis switches between various hosts in its habitat, depending on their abundance and accessibility. Because it is a generalist and its hosts are probably hard to find, G. agilis has compensated by reducing costly investment in germinal tissue (eggs) for somatic tissue (maintenance) that has resulted in a longer life-span. On the other hand, L. nana has evolved a reproductive strategy that is intimately associated with fully cocooned pupae of gregarious parasitoids in the genus Cotesia (Schwarz and Shaw 2000). Because it has wings and can fly efficiently, L. nana is able to exploit a much larger area than G. agilis, a factor that has allowed it to specialize but at the cost of a reduced adult life-span. Consequently, reproduction is more "frontloaded" in L. nana than in G. agilis, and it is able to mobilize fairly large numbers of eggs in a short time, enabling it to exploit full broods of its host.

Pre-pupae and pupae of $C$. glomerata are attacked by at least five species of secondary hyperparasitoids in the Netherlands (Harvey, unpublished). In addition to differences in host specialization, niche differentiation amongst the various species appears to be influenced by the architectural complexity of the plant as well as on the structure of the surrounding plant community on which $P$. brassicae and $C$. glomerata interact. For example, winged hyperparasitoids like L. nana and a closely related species Acrolyta nens are much more effective at foraging for host cocoons located higher up on the food plant, whereas wingless species like G. agilis are more restricted to lower parts of the plant. A recent study (unpublished) also found that both $A$. nens and $L$. nana emerged from single cocoon broods of $C$. glomerata in plots of B. nigra plants. Future experiments with A. nens and L. nana will hopefully reveal the extent to which the reproductive biologies and ecologies of these hyperparasitoids are convergent or if each species possesses slightly different adaptations that enable both to exploit hosts with equal efficiency in nature.

Acknowledgments The author thanks Leo Koopman and André Gidding of Wageningen University for a constant supply of herbivores and parasitoids; Leontien Witjes and Roel Wagenaar for help with experiments; and Martijn Bezemer for his comments on an earlier draft of the manuscript.

\section{References}

Bai B, Smith SM (1993) Effect of host availability on reproduction and survival of the parasitoid wasp, Trichogramma minutum. Ecol Entomol 18:279-286

Bezant ET (1956) Gelis micrurus Forster (Hymenoptera: Ichneumonidae) parasitising a lycosid spider egg sac. Ent Mon Mag 92:106

Black AR, Dodson SI (1990) Demographic costs of Chaoborus induced phenotypic plasticity in Daphnia pulex. Oecologia 83:117-122

Boggs CL (1997) Reproductive allocation from reserves and income in butterfly species with differing adult diets. Ecology 78:181-191 
Bonsall MB, Jansen VAA, Hassell MP (2004) Life history trade-offs assemble ecological guilds. Science 306:111-114

Chan MS, Godfray HCJ (1993) Host-feeding strategies of parasitoid wasps. Evol Biol 7:593-604

Cobb LM, Cobb VA (2004) Occurrence of parasitoid wasps, Baeus sp. and Gelis sp., in the egg sacs of the wolf spiders Pardosa moesta and Pardosa sternalis (Araneae, Lycosidae) in southeastern Idaho. Can Field Nat 118:122-123

Collier TR (1995) Adding physiological realism to dynamic state variable models of parasitoid host feeding. Evol Ecol 9:217-234

Ellers J (1996) Fat and eggs, an alternative method to measure the trade-off between survival and reproduction in insect parasitoids. Neth J Zool 46:227-235

Ellers J, Jervis M (2003) Body size and the timing of egg reproduction in parasitoid wasps. Oikos 102:164172

Ellers J, Van Alphen JJM, Sevenster JG (1998) A field study of size-fitness relationships in the parasitoid Asobara tabida. J Anim Ecol 67:318-324

Ellers J, Sevenster JG, Driessen G (2000) Egg load evolution in parasitoids. Am Nat 156:650-665

Giron D, Rivero A, Mandon N, Darrouzet E, Casas J (2002) The physiology of host-feeding in parasitic wasps: implications for survival. Funct Ecol 16:750-757

Giron D, Pincebourde S, Casas J (2004) Lifetime gains of host-feeding in a synovigenic parasitic wasp. Physiol Entomol 29:436-442

Godfray HCJ (1994) Parasitoids: behavioral and evolutionary ecology. Princeton University Press, Princeton, NJ

Harvey JA (2000) Dynamic effects of parasitism by an endoparasitoid wasp on the development of two host species: implications for host quality and parasitoid fitness. Ecol Entomol 25:267-278

Harvey JA, Harvey IF, Thompson DJ (2001) Lifetime reproductive success in the endoparasitoid wasp, Venturia canescens. J Insect Behav 14:573-593

Harvey JA, Strand MR (2002) The developmental strategies of endoparasitoid wasps vary with host feeding ecology. Ecology 83:2349-2451

Harvey JA, Witjes LMA (2005) Comparing and contrasting life-history and development strategies in the pupal hyperparasitoids Lysibia nana and Gelis agilis (Hymenoptera: Ichneumonidae). Appl Entomol Zool 40:309-316

Harvey JA, Vet LEM, Witjes LMA, Bezemer TM (2006) Remarkable similarity in body mass of a secondary hyperparasitoid Lysibia nana and its primary parasitoid host Cotesia glomerata emerging from cocoons of comparable size. Arch Insect Biochem Physiol 61:170-183

Heimpel GH, Collier TR (1996) The evolution of host-feeding behavior in insect parasitoids. Biol Rev 71:373-400

Heimpel GE, Rosenheim JA, Kattari DD (1997) Adult feeding and lifetime reproductive success in the parasitoid Aphytis melinus. Entomol Exp Appl 83:305-315

Hohmann CL, Luck RF, Oatman ER, Platner GR (1989) Effects of different biological factors on longevity and fecundity of Trichogramma platneri Nagarkatti (Hymenoptera: Trichogrammatidae). An du Soc Entomol Brasil 18:61-70

Holomuzki JR, Biggs BJF (2006) Habitat-specific variation and performance trade-offs in shell armature of New Zealand mudsnails. Ecology 87:1038-1047

Jervis MA, Kidd NAC (1986) Host feeding strategies in hymenopteran parasitoids. Biol Rev 61:395-434

Jervis MA, Heimpel GE, Ferns PN, Harvey JA, Kidd NAC (2001) Life-history strategies in parasitoid wasps: a comparative analysis of "ovigeny". J Anim Ecol 70:442-458

Lauziere I, Perez-Lachaud G, Brodeur J (2000) Effect of female body size and adult feeding on the fecundity and longevity of the parasitoid Cephalonomia stephanoderis Betrem (Hymenoptera: Bethylidae). Ann Entomol Soc Am 93:103-109

Lessells CR (1991) The evolution of life histories. In: Davies NB, Krebs JR (eds) Behavioural ecology: an evolutionary approach. Blackwell, Oxford, pp 32-68

Levins R (1966) The strategy of model building in population biology. Am Sci 54:421-431

Mackauer M, Sequeira R (1993) Patterns of development in insect parasites. In: Beckage NE, Thompson SN, Federici BA (eds) Parasites and pathogens of insects. Academic, New York, pp 1-23

Orr DB, Boethel DJ (1990) Reproductive potential of Telenomus cristatus and T. podisi (Hymenoptera: Scelionidae), two egg parasitoids of pentatomids (Heteroptera). Ann Entomol Soc Am 83:902-905

Pexton JJ, Mayhew PJ (2002) Siblicide and life-history evolution in parasitoids. Behav Ecol 13:690-695

Price PW (1972) Parasitoids utilizing the same host: adaptive nature of differences in size and form. Ecology 53:190-195

Reznick D (1985) Costs of reproduction: an evaluation of the empirical evidence. Oikos 44:257-267

Riessen HP (1992) Cost-benefit model for the induction of an antipredator defense. Am Nat 140:349-362 
Rivero A, West SA (2005) The costs and benefits of host feeding in parasitoids. Anim Behav 69:1293-1301

Roff DA (1992) The evolution of life histories, theory and analysis. Chapman \& Hall, New York

Schwarz M, Boriani M (1994) Redescription of Gelis longulus (Hymenoptera, Ichneumonidae), a parasitoid of Ocnerostoma piniarielum (Lepidoptera: Yponomeutidae). Eur J Entomol 91:331-334

Schwarz M, Shaw MR (2000) Western Palearctic Cryptinae (Hymenoptera: Ichneumonidae) in the National Museums of Scotland, with nomenclatural changes, taxonomic notes, rearing records and special reference to the British check list. Part 3. Tribe Phygadeuontini, subtribes Chiroticina, Acrolytina, Hemitelina and Gelina (excluding Gelis), with descriptions of new species. Entomol Gaz 51:147-186 Stearns SC (1992) The evolution of life histories. Oxford University Press, Oxford, UK

Toth P, Lucas J (2005) Parasitic Ichneumonoidea on the horse chestnut leaf miner, Cameraria ohridella (Lepidoptera: Gracillariidae) in Slovakia. J Pest Sci 78:151-154

Ueno T (1999) Reproduction and host-feeding in the solitary parasitoid wasp Pimpla nipponica (Hymenoptera: Ichneumonidae). Invert Rep Dev 35:231-237

van Baarlen P, Topping CJ, Sunderland KD (1996) Host location by Gelis festinans, an eggsac parasitoid of the linyphiid spider Erigone atra. Entomol Exp Appl 81:155-163

van Noordwijk AJ, de Jong G (1986) Acquisition and allocation of resources: their influence on variation in life-history tactics. Am Nat 128:137-142

van Nouhuys S, Hanski I (2000) Apparent competition between parasitoids mediated by a shared hyperparasitoid. Ecol Lett 3:82-84

Vinson SB, Iwantsch GF (1980) Host regulation by insect parasitoids. Q Rev Biol 55:143-165

Vollrath F, Parker GA (1992) Sexual dimorphism and distorted sex ratios in spiders. Nature 360:156-159

Wäckers FL (2001) A comparison of nectar- and honeydew sugars with respect to their utilization by the hymenopteran parasitoid Cotesia glomerata. J Insect Physiol 47:1077-1084 